\title{
Impact of PV for Self-consumption in the Day-ahead Spot Prices
}

\author{
F.J. Soares ${ }^{1}$ and J.P. Iria ${ }^{1,2}$ \\ ${ }^{1}$ INESC TEC and ${ }^{2}$ FEUP \\ Porto, Portugal \\ fsoares@inesctec.pt, jpiria@inestec.pt
}

\author{
J.C. Sousa ${ }^{3}$, V. Mendes ${ }^{3}$ and A. C. Nunes ${ }^{3}$ \\ ${ }^{3}$ EDP- Gestão da Produção de Energia \\ Porto, Portugal \\ jose.sousa@edp.pt, virgilio.mendes@edp.pt, \\ anacristina.nunes@edp.pt
}

\begin{abstract}
This paper presents an analysis of the impacts of photovoltaics and storage units for self-consumption in the dayahead spot prices. A methodology is proposed, to access these impacts in the Iberian electricity market for 2015, 2020 and 2030.
\end{abstract}

Index Terms-Electricity markets, storage, photovoltaic panels, self-consumption

\section{INTRODUCTION}

The gradual decrease in the cost of photovoltaic (PV) systems has contributed to increase consumer interest in this type of technology [1]. The economic viability of using these systems for self-consumption, considering retailers' electricity rates as basis of comparison, will certainly contribute to a significant increase in PV integration over the coming years.

The large scale integration of these systems in electrical networks will not only have a significant impact in the greenhouse gas emissions associated with the production of electricity, but also in the demand profiles and in markets' spot prices. If the integration of PV systems is made together with batteries, the impacts are expected to be even higher.

Several publications exist focusing on the impacts of PV at household level, as well as at distribution network level. In [2], for example, the authors presented the results of integrating systems with PV in 30 households with different energy demand profiles. Despite the analysis made to the impacts on the aggregated demand profiles, no evaluation was made at nationwide scale. Other works, such as [3], analyzed the impacts of PV integration on the spot prices, but they did not consider the joint integration of PV and storage.

The aim of this paper is to access the impact of PV and storage for self-consumption in the day-ahead spot prices. To do that a methodology to quantify the alterations in the load diagram was developed. In addition, a market simulation tool is used to access the evolution of the wholesale electricity prices. The Portuguese system and the Iberian market (MIBEL) were used as test cases for 2015, 2020 and 2030.

The paper is organized in five sections. After a brief introduction in section I, the methodology developed to access the evolution of the load diagram is described in section II. The market simulation tool is described in section III. The results achieved are presented in section IV and the conclusions drawn are described in $\mathrm{V}$.

The work of F.J. Soares and J.P. Iria was supported by the SusCity project ("SusCity -MITPTB/CS/0026/2013") financed by Portuguese funds through Fundação para a Ciência e Tecnologia (FCT). The work of J.P. Iria was also supported by FCT Ph.D. Scholarship PD/BD/113716/2015.

\section{Methodology Used to ACCESS THE EVOLUTION OF THE LOAD DIAGRAM}

The methodology developed is a bottom-up approach that considers the realization of three distinct studies:

1) Forecasting of the Portuguese load diagram;

2) Accessing the impact of PV integration for selfconsumption in the load diagram;

3) Accessing the impact of PV and storage integration in the load diagram.

The tasks were based on data from 2015 and were performed considering a time resolution of 1 hour for 2015, 2020 and 2030. The studies were carried out for three different regions of Portugal - North, Centre and South - in order to consider locations with different latitudes and thus with different insolation levels.

The methodology is briefly presented in Figure 1. More details about it are provided in the following subsections.

\section{A. Forecasting of the Portuguese Demand Profile - Study 1)}

The first step for the calculation of the aggregated load profile was the collection of typical hourly consumption patterns for individual clients. This data was obtained from the Portuguese Energy Services Regulator (ERSE) [4]. Two typical profiles for Low Voltage (LV) and one for Medium, High and Very High Voltage clients (MV, HV and VHV) were considered.

The representativeness of each profile, as well as the average daily energy consumption for 2015 is shown in Table I and is also provided by ERSE in [5].

TABLE I

Characteristics of the Portuguese consumers in 2015

\begin{tabular}{llll}
\hline \hline $\begin{array}{l}\text { Type of } \\
\text { consumer }\end{array}$ & $\begin{array}{l}\text { Annual } \\
\text { consumption } \\
\text { (GWh) }\end{array}$ & $\begin{array}{l}\text { Number } \\
\text { of clients }\end{array}$ & $\begin{array}{l}\text { Average daily } \\
\text { consumption per } \\
\text { client }(\mathrm{kWh})\end{array}$ \\
\hline LV-A & 19006 & 6022235 & 8.65 \\
\hline LV-B & 3304 & 33550 & 270 \\
\hline MV & 13636 & 23575 & 1585 \\
\hline HV & 6395 & 283 & 61910 \\
\hline VHV & 2192 & 69 & 87036 \\
\hline \hline
\end{tabular}

The Portuguese aggregated load profile is obtained by multiplying the individual profiles by the respective number of clients and then sum them all. Afterwards, the losses due to 
electricity transmission and distribution are also added to the aggregate load profile, since this energy is also traded in the day-ahead market.

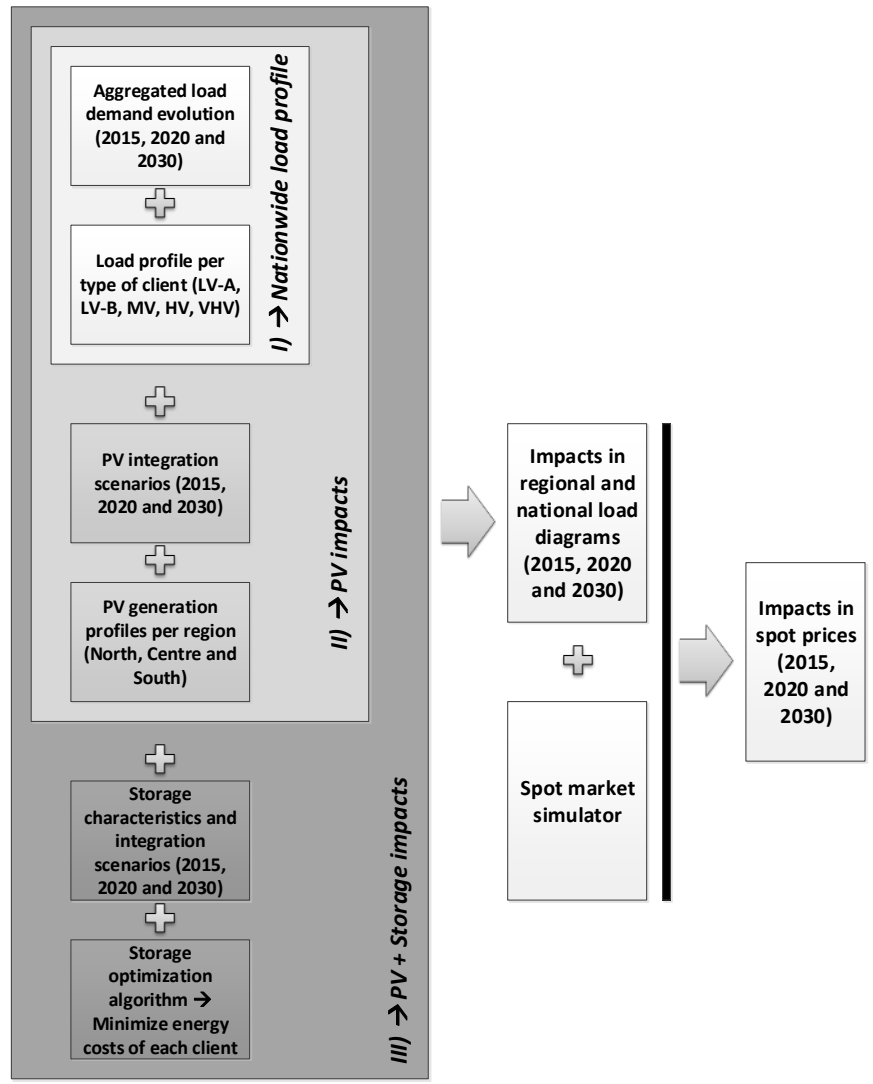

Figure 1. Methodology overview.

The demand evolution considered is shown in Figure 2. The decrease in consumption registered between 2010 and 2014 was largely due to the economic crisis. With the slight recovery of the Portuguese economy and with the gradual integration of electric vehicles, a progressive increase in the electricity consumption is expected until 2030.

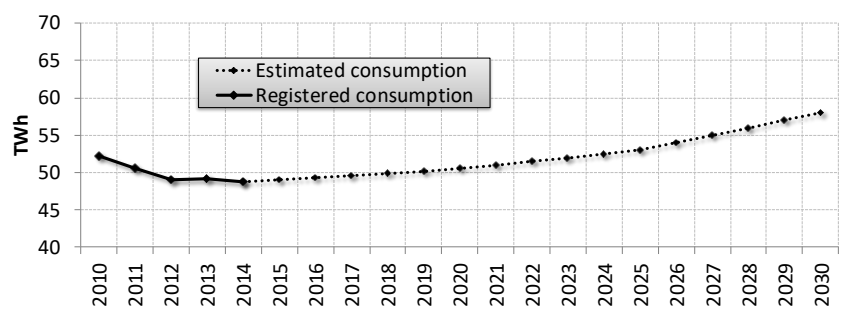

Figure 2. Evolution of the energy traded in the MIBEL, concerning Portugal.

\section{B. Impacts of PV for Self-consumption - Study 2)}

As shown in Figure 1, the impacts of the PV for selfconsumption were assessed by subtracting the energy generated by all the PV installations considered to the aggregated demand profile obtained in subsection A.

The number of PV installations assumed to exist in each scenario are presented in Table II. The values presented in the last column II were obtained using typical PV generation profiles for the insulation levels and clear-sky indexes registered in the three regions of Portugal considered - North, Centre and South.

TABLE II

Number of PV installations for self-consumption in 2015, 2020 and 2030

\begin{tabular}{|c|c|c|c|c|}
\hline Year & $\begin{array}{l}\text { Type of } \\
\text { consumer }\end{array}$ & $\begin{array}{l}\% \text { of } \\
\text { consumers } \\
\text { with PV }\end{array}$ & $\begin{array}{l}\text { Power } \\
\text { per PV } \\
\text { unit }(\mathrm{kW})\end{array}$ & $\begin{array}{l}\text { Average energy } \\
\text { generated per year } \\
\text { (GWh) }\end{array}$ \\
\hline \multirow{3}{*}{2015} & LV-A & 0.02 & 0.5 & \multirow{3}{*}{11.2} \\
\hline & LV-B & 0.16 & 16.7 & \\
\hline & MV & 0.22 & 48.9 & \\
\hline \multirow{3}{*}{2020} & LV-A & 0.61 & 0.5 & \multirow{3}{*}{173.1} \\
\hline & LV-B & 2.22 & 16.7 & \\
\hline & MV & 2.63 & 48.9 & \\
\hline \multirow{3}{*}{2030} & LV-A & 9.18 & 0.5 & \multirow{3}{*}{1088.1} \\
\hline & LV-B & 9.95 & 16.7 & \\
\hline & MV & 9.95 & 48.9 & \\
\hline
\end{tabular}

The power per PV unit was determined taking into account the regulation currently applied in Portugal for selfconsumption. This is regulated by the Decree-Law 153/2014 that entered into force in October 2014 [6]. The most relevant aspects considered are presented next:

- The energy generated by the PV installation can be consumed locally. If generation is higher that the demand, the energy in excess can be injected in the network (article 7th);

- To the extent possible, the PV installation shall be designed to match generation with local demand (article 8th);

- When generation is higher than demand, if the energy in excess is injected in the network, the remuneration rate is $90 \%$ of the wholesale market price (article 24th).

\section{Optimization of the Storage Operation - Study 3)}

This section describes the multi-period algorithm used to optimize the operation of the storage units installed at low and medium voltage levels. Its main objective is to minimize the cost of each consumer buying energy, taking into account the legislation currently into force for self-consumption in Portugal [6]. The optimization model is given by:

$$
\operatorname{Min} \sum_{t=t_{0}}^{T}\left\{\begin{array}{r}
p_{t}\left(E_{t}^{C}-E_{t}^{D}+E_{t}^{L}-E_{t}^{P V}\right), E_{t}^{C}-E_{t}^{D}+E_{t}^{L}-E_{t}^{P V} \geq 0 \\
-r_{t}\left(E_{t}^{C}-E_{t}^{D}+E_{t}^{L}-E_{t}^{P V}\right), E_{t}^{C}-E_{t}^{D}+E_{t}^{L}-E_{t}^{P V}<0
\end{array}\right.
$$

subject to:

$$
\begin{array}{ll}
0 \leq \frac{E_{t}^{D}}{\Delta t} \leq \partial_{t}^{D} P^{\text {Max }}, & \forall t \in\left\{t_{0}, \ldots, T\right\} \\
0 \leq \frac{E_{t}^{C}}{\Delta t} \leq \partial_{t}^{C} P^{\text {Max }}, & \forall t \in\left\{t_{0}, \ldots, T\right\} \\
\partial_{t}^{D}+\partial_{t}^{C}<2, & \forall t \in\left\{t_{0}, \ldots, T\right\} \\
C_{t+1}=C_{t}-E_{t}^{D}(1+(1-\eta))+E_{t}^{C} \eta, & \forall t \in\left\{t_{0}, \ldots, T\right\} \\
C^{\text {Min }} \leq C_{t+1} \leq C^{\text {Max }}, & \forall t \in\left\{t_{0}, \ldots, T\right\}
\end{array}
$$

The objective function has two terms. The first term is the cost of buying electricity. The second term corresponds to the remuneration received by the end user due to inject energy in the grid. The decision variables are $E_{t}^{C}$ and $E_{t}^{D}$, which represent the electrical energy charged and discharged from the battery in time interval $t$. The parameters in (1) are as follows: $E_{t}^{L}$ is 
the electrical energy consumed; $E_{t}^{P V}$ is the electrical energy produced by $\mathrm{PV} ; r_{t}$ is the remuneration price paid to the consumers due to the injection of energy in electricity grid; $p_{t}$ is the electricity retailing price; and $T$ is the number of optimization periods (in this case, $T=24$ periods of 1 hour).

The model has five constraints. The first constraint is (2) and ensures that the electric power of the battery does not exceed its maximum discharging power when the battery is in discharging mode $\left(\partial_{t}^{D}=1\right) . \Delta t$ is the duration of the time interval $t$ (1 hour). The second constraint is (3) and guarantees that the maximum charging power is not exceeded when the battery is in charging mode $\left(\partial_{t}^{C}=1\right)$. The third constraint is (4) and is used to guarantee that the battery does not work in charging and discharging mode at same time. $\partial_{t}^{D}$ and $\partial_{t}^{C}$ are binary auxiliary variables used to define the battery operation mode.

The fourth constrain is (5) and sets the energy stored in the battery $\left(C_{t+1}\right) . \eta$ is the efficiency of the battery charging and discharging process. The fifth constrain is (6) and ensures that the energy stored in the battery is between the minimum and maximum possible values $\left(C^{\text {Min }}-C^{\text {Max }}\right)$.

The objective function (1) can be transformed into a mixed integer linear problem using the mathematical techniques described in [7]. Therefore, the full formulation becomes:

$$
\operatorname{Min} \sum_{t=t_{0}}^{T} p_{t} s_{t}^{1}-r_{t} s_{t}^{2}+N\left(\partial_{t}^{D}+\partial_{t}^{C}\right)
$$

subject to:

$$
\begin{array}{ll}
s_{t}^{1}-s_{t}^{2}=E_{t}^{C}-E_{t}^{D}+E_{t}^{L}-E_{t}^{P V}, & \forall t \in\left\{t_{0}, \ldots, T\right\} \\
s_{t}^{1} \leq \emptyset_{t} M, & \forall t \in\left\{t_{0}, \ldots, T\right\} \\
s_{t}^{2} \leq\left(1-\emptyset_{t}\right) M, & \forall t \in\left\{t_{0}, \ldots, T\right\} \\
s_{t}^{1}, s_{t}^{2} \geq 0, & \forall t \in\left\{t_{0}, \ldots, T\right\} \\
0 \leq \frac{E_{t}^{D}}{\Delta t} \leq \partial_{t}^{D} P^{\text {Max }}, & \forall t \in\left\{t_{0}, \ldots, T\right\} \\
0 \leq \frac{E_{t}^{C}}{\Delta t} \leq \partial_{t}^{C} P^{\text {Max }}, & \forall t \in\left\{t_{0}, \ldots, T\right\} \\
\partial_{t}^{D}+\partial_{t}^{C}<2, & \forall t \in\left\{t_{0}, \ldots, T\right\} \\
C_{t+1}=C_{t}-E_{t}^{D}(1+(1-\eta))+E_{t}^{C} \eta, & \forall t \in\left\{t_{0}, \ldots, T\right\} \\
C^{\text {Min }} \leq C_{t+1} \leq C^{\text {Max }}, & \forall t \in\left\{t_{0}, \ldots, T\right\}
\end{array}
$$

where $s_{t}^{1}$ and $s_{t}^{2}$ are decision variables used to represent consumption and injection, from the distribution grid perspective. The binary variable $\emptyset_{t}$ and the large positive number $M$ are used in (9) and (10) to impose one of the two constraints. The term $N\left(\partial_{t}^{D}+\partial_{t}^{C}\right)$ is added to the objective function with the objective of penalizing unnecessary charging and discharging cycles. $N$ is a small positive number.

Three types of time-of-use prices were adopted, i.e. two for LV consumers with three price periods in the cold months and four in hot months, and one for MV consumers with four price periods during the entire year. Further details about the timeof-use tariffs used are available in [8].

Regarding storage, lithium-ion batteries were assumed to be chosen by clients with PV installations. Table III presents the characteristics of the storage units. The capacity of the batteries was defined assuming that it should be capable of absorbing all the excess energy generated by the PV system.

\section{TABLE III}

CHARACTERISTICS OF THE LITHIUM-ION BATTERIES ADOPTED

\begin{tabular}{llll}
\hline \hline Type of consumer & LV-A & LV-B & MV \\
\hline$C^{\text {Max }}(k W h)$ & 1 & 20 & 50 \\
\hline$C^{\text {Min }}(k W h)$ & 0.1 & 2 & 5 \\
\hline$\eta$ & 0.9 & 0.9 & 0.9 \\
\hline$P^{\text {Max }}(k W)$ & 0.35 & 6 & 15 \\
\hline \hline
\end{tabular}

The number of storage units assumed to be equal to the number of PV systems for all scenarios.

The individual load profiles were computed by adding the individual diagrams calculated in section B to the charging and discharging profiles of the storage units. Afterwards, the Portuguese aggregated load profile is obtained by multiplying the individual profiles by the respective number of clients and then sum them all.

\section{MARKET SimUlation TOOL}

The impact of the aggregated load profiles in the day-ahead electricity prices was computed using the market simulator, EMCAS model.

EMCAS (Electricity Market Complex Adaptive Systems) is a commercial Agent Based Model software, developed by the Argonne National Lab, which has the capability of taking decentralized decisions based on self-learning and selfadaptive agents [9]. EMCAS uses an agent-based modeling approach to simulate the operation of liberalized electricity markets. Market participants are represented as autonomous agents that have their own objectives, decision-making rules, and behavioral patterns. Agents are modeled as independent entities that make decisions and take actions using the available information, sometimes limited and uncertain, similar to how organizations and companies operate in the real world. Some of the capabilities of EMCAS include the simulation of day-ahead and real-time markets, modeling of take-or-pay fuel contracts, bilateral financial contracts, demand response, carbon emissions and allowances, among others [9]. EMCAS can also handle congestion management either through locational marginal pricing or system marginal price and uplift charges. The hydro power plants are modeled as price takers and bid their supply offers at zero price. In the basic implementation, most of the hydro generation will be placed in hours with the highest projected prices.

EMCAS is linked to VALORAGUA model [10] that provides long-term operation planning strategies for hydro plants. With this information, EMCAS uses the price forecasts and weekly hydro schedules given by VALORAGUA to do intra-week hydro plant optimization for hourly supply offers. VALORAGUA is a hydro thermal model that has been in use for several decades as a hydro-thermal coordination model with the objective of optimizing overall system operation over a period of up to 1 year. The model optimizes the operation of hydro and pumped-storage power plants and management of reservoirs and hydro cascades. The objective function minimizes the overall system operating costs based on the calculated expected value of water in each time period. This 
model takes into account system configuration, demand, thermal and renewable capacity, reservoir characteristics, hydro cascades, and historical water inflows and generates weekly schedules for each of the hydro power plants based on stochastic dynamic programming and non-linear programming algorithms.

\section{RESULTS}

\section{A. Individual Profiles}

This section presents an example of individual load profiles obtained for LV and MV clients, as shown in Figures 3, 4 and 5. Three scenarios are depicted, as follows: a base case, where only consumption is considered (Load); the second scenario results from adding PV generation to the base case (Load+PV); and the third scenario consists in optimizing the load profile, considering a storage and a PV unit (Load+PV+Storage).

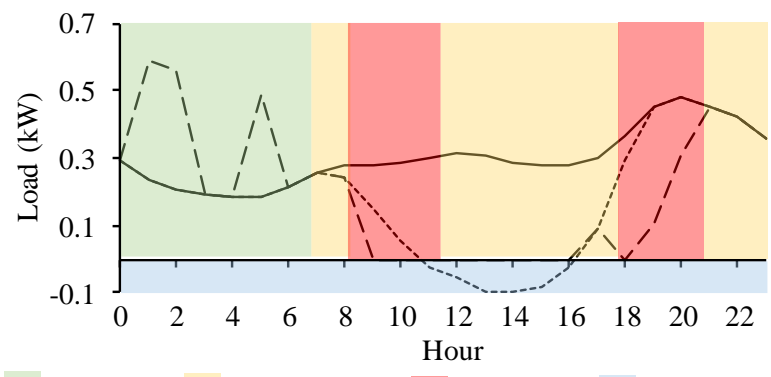

Low prices Medium prices High prices Rem. prices

Load ------- Load+PV - - - Load+PV+Storage

Figure 3. Load profile of a LV-A client located in the centre of Portugal for one day of March 2015.

In a self-consumption perspective, the PV unit is sized, in order to avoid the injection of electrical energy in the distribution grid. However, there are periods when that injection cannot be avoided, as shown in Figure 3 in the period between 11 and $16 \mathrm{~h}$.

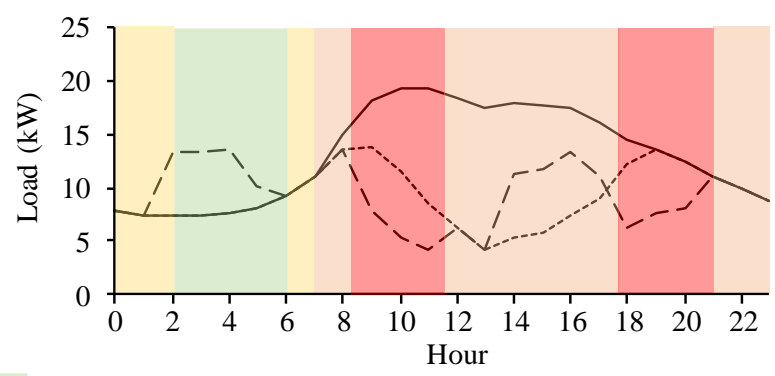

Low prices Medium prices High prices Very high pr.

L Load -----.. Load+PV - - - Load+PV+Storage

Figure 4. Load profile of a LV-B client located in the centre of Portugal for one day of March 2015.

The optimization of the load profile in a self-consumption perspective, considering a storage unit follows the remuneratory rules of the Portuguese legislation. Figures 3, 4 and 5 show that the battery absorbs electrical energy in the periods of low prices, and injects it back in the grid in the periods of high prices. In the periods where an excess of PV generation exists, the storage unit absorbs the excess of electrical energy, in order to inject in the periods of high prices, as shown in Figure 3. Injecting electrical energy in the distribution grid is disadvantageous for the consumer, since the remuneration prices in Portugal are lower than retailing prices. This optimization strategy allows reducing the electricity costs of the end user.

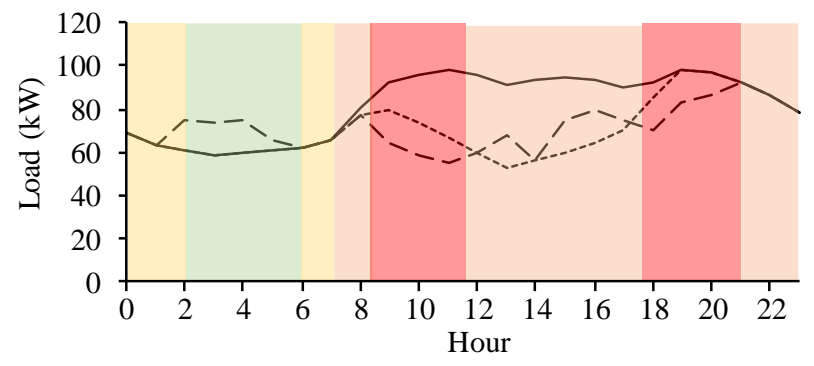

Low prices Medium prices High prices Very high pr.

— Load -------- Load+PV - - - L Load+PV+Storage

Figure 5. Load profile of a MV client located in the centre of Portugal for one day of March 2015.

\section{B. Aggregated Profiles}

The aggregated load profiles computed for one day of March in 2015, 2020 and 2030 are presented in Figures 6, 7 and 8, respectively. These figures show that the increase of PV and storage integration along the years have impact in the Portuguese load profile.

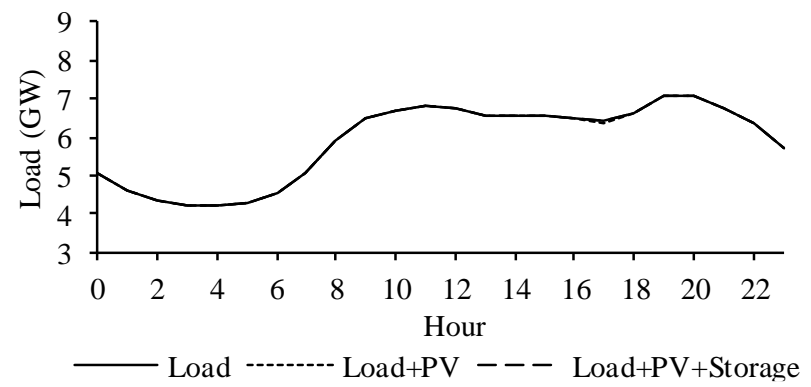

Figure 6. Aggregated load profiles of Portugal for one day of March 2015.

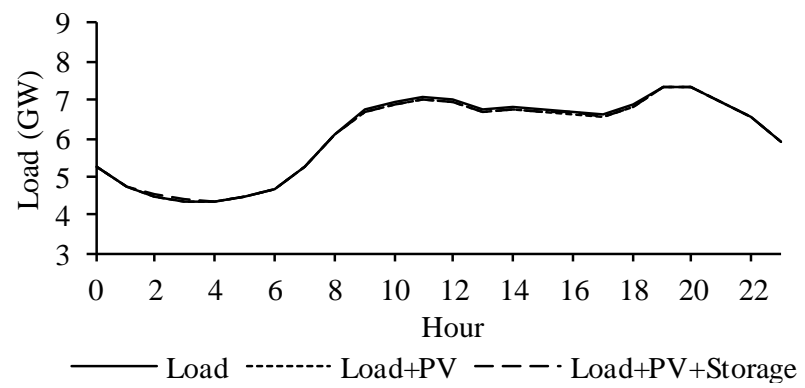

Figure 7. Aggregated load profiles of Portugal for one day of March 2020.

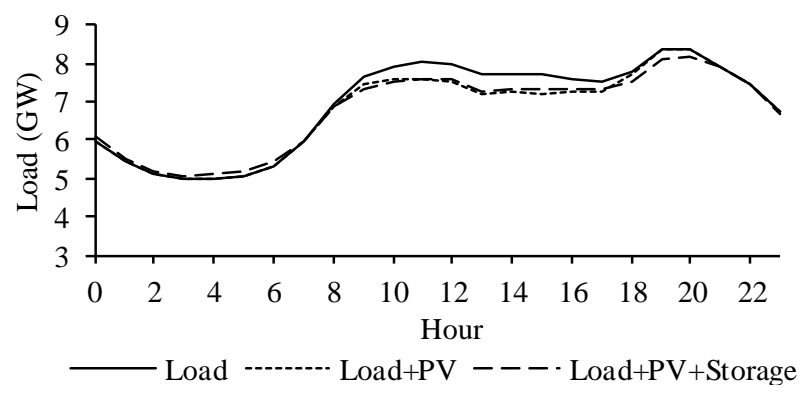

Figure 8. Aggregated load profiles of Portugal for one day of March 2030. 


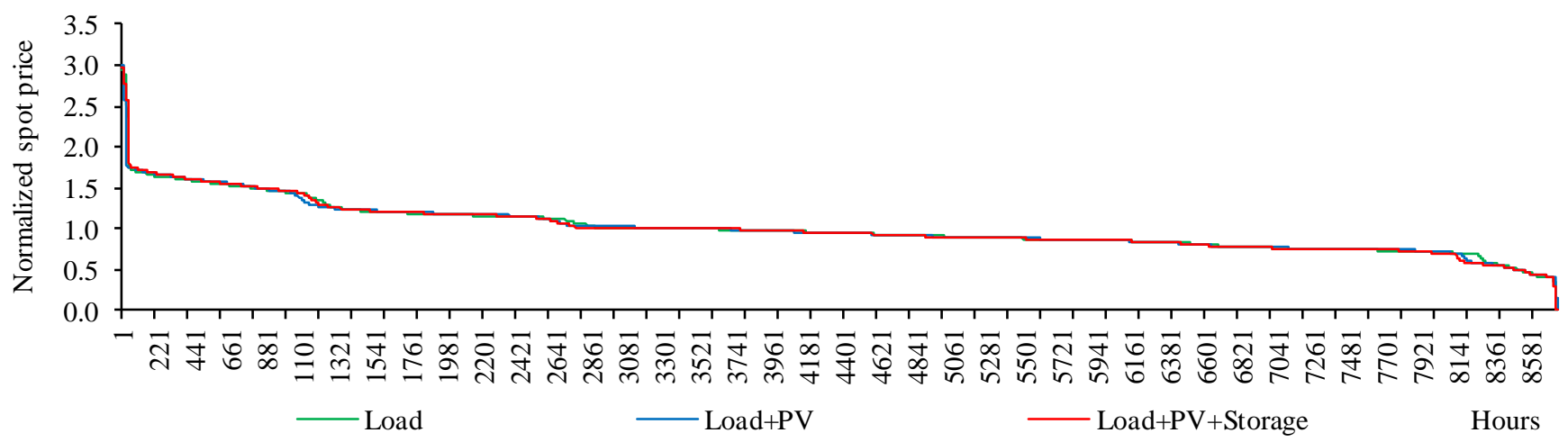

Figure 9. Normalized spot prices, based on the annual average price for 2030.

The integration of PV decreases the net load in the periods of high generation. Furthermore, the joint deployment of storage units with PV also increases the net load in the valley hours, due to the low retailing prices in that hours. This effect is shown in Figure 8.

\section{Impact in the Day-ahead Spot Prices}

The impacts in the day-ahead electricity prices due to the integration of PV and storage units are presented in Table IV. The results show that the integration of PV for selfconsumption allows to reduce the average spot price in comparison with the base case (only Load). The integration of $\mathrm{PV}$ reduces the total system load, which avoids the dispatch of more expensive generators.

The joint integration of $\mathrm{PV}$ and storage accentuates the decrease of the average spot price, since reduces simultaneously the total system load and the load in the offpeak periods. The storage units allow to shift electric consumption from peak to off-peak periods.

TABLE IV

REDUCTION OF THE AVERAGE SPOT PRICE DUE TO THE INTEGRATION OF PV AND STORAGE UNITS. COMPARISON WITH A BASE CASE (ONLY LOAD).

\begin{tabular}{llll}
\hline \hline Year & 2015 & 2020 & 2030 \\
\hline Load+PV vs. Load & $-0.2 \%$ & $-1.6 \%$ & $-1.7 \%$ \\
\hline Load+PV+Storage vs. Load & $-1.3 \%$ & $-2.0 \%$ & $-2.3 \%$ \\
\hline \hline
\end{tabular}

Figure 9 presents the normalized spot prices for 2030. The spot prices were normalized, based on the annual average spot price. These curves allow understanding if significant variations in the electricity prices are foreseen. These variations can be important, for instance, to plan different bidding strategies for hydro power plants with pumped storage. In this case, the results do not show significant variations in the day-ahead spot prices for 2030 .

It is important to stress that only the impact of integrating PV and storage units in the Portuguese side was studied, i.e. the integration in the Spanish side was not considered.

\section{CONCLUSIONS}

This paper presented an analysis of the impacts of introducing PV and storage units for self-consumption in the Portuguese load profile and in the MIBEL electricity prices for 2015, 2020 and 2030. The results shown that the increase integration of PV leads to the reduction of the annual average spot price. This price reduction is accentuated, when storage is coupled with PV.

Future work consists in analyzing the impact of flexible loads in the electricity spot prices. Initial works in this area show that the impacts can be significant [11].

\section{ACKNOWLEDGMENT}

The authors gratefully acknowledge Carlos Silva, Maria Rodrigues and Filipa Amorim from Instituto Superior Técnico (IST) for providing predictions regarding the deployment of PV in Portugal, as well as time series of PV generation.

\section{REFERENCES}

[1] International Energy Agency (IEA), "Technology Roadmap - Solar Photovoltaic Energy", 2014.

[2] P. Balcombe, D. Rigby, A. Azapagic, "Energy self-sufficiency, grid demand variability and consumer costs: Integrating solar PV, Stirling engine CHP and battery storage," Applied Energy, vol. 155, pp. 393-408, Oct. 2015

[3] P. Vithayasrichareon, J. Riesz, I. MacGill, "Impact of high variable renewable generation on future market prices and generator revenue," in Power and Energy Engineering Conference (APPEEC), 2015 IEEE PES Asia-Pacific, pp.1-5, 15-18 Nov. 2015.

[4] ERSE, "Perfis de perdas, perfis de consumo e perfis de produção", 2015. (in Portuguese)

[5] ERSE, “Caracterização da procura de energia eléctrica em 2015”, 2015. (in Portuguese)

[6] Decree Law ${ }^{\circ} 153 / 2014$, Portuguese legislation on self-consumption, Oct. 2014 (in Portuguese).

[7] J. Bisschop. "AIMMS optimization modeling," Paragon Decision Technology, 1999. Available: http://www.aimms.com

[8] ERSE, "Tarifas e Preços para a energia eléctrica e outros serviços em 2015 e parâmetros para o Período de regulação 2015-2017", 2015. (in Portuguese) Available: http://www.erse.pt/pt/electricidade/tarifaseprecos/2015/Documents/Pag inaPrincipal/Tarifas\%20e\%20Pre\%C3\%A7os\%202015.pdf

[9] P. Thimmapuram, T. D. Veselka, V. Koritarov, S. Vilela, R. Pereira, R. F. Silva, "Modeling hydro power plants in deregulated electricity markets: Integration and application of EMCAS and VALORAGUA", in Proc. EEM2008, pp. 1-6, May 2008.

[10] Electricidade de Portugal (EDP), "VALORAGUA, A Model for the Optimal Management of a Hydro Thermal Electric Power System," 1987.

[11] J.P. Iria, F.J. Soares, R.J. Bessa, "Optimized Demand Response Bidding in the Wholesale Market under Scenarios of Prices and Temperatures," in PowerTech, 2015 IEEE Eindhoven, vol., no., pp.1-6, 2015. 